\title{
Simulations of Contrail Optical Properties and Radiative Forcing for Various Crystal Shapes
}

\author{
KRZYSZTOF M. MARKOWICZ \\ Institute of Geophysics, Faculty of Physics, University of Warsaw, Warsaw, Poland \\ MARCIN L. WITEK \\ California Institute of Technology, Pasadena, California
}

(Manuscript received 9 August 2010, in final form 1 April 2011)

\begin{abstract}
The aim of this study is to investigate the sensitivity of radiative-forcing computations to various contrail crystal shape models. Contrail optical properties in the shortwave and longwave ranges are derived using a ray-tracing geometric method and the discrete dipole approximation method, respectively. Both methods present good correspondence of the single-scattering albedo and the asymmetry parameter in a transition range $(3-8 \mu \mathrm{m})$. There are substantial differences in single-scattering properties among 10 crystal models investigated here (e.g., hexagonal columns and plates with different aspect ratios, and spherical particles). The single-scattering albedo and the asymmetry parameter both vary by up to 0.1 among various crystal shapes. The computed single-scattering properties are incorporated in the moderate-resolution atmospheric radiance and transmittance model (MODTRAN) radiative transfer code to simulate solar and infrared fluxes at the top of the atmosphere. Particle shapes have a strong impact on the contrail radiative forcing in both the shortwave and longwave ranges. The differences in the net radiative forcing among optical models reach $50 \%$ with respect to the mean model value. The hexagonal-column and hexagonal-plate particles show the smallest net radiative forcing, and the largest forcing is obtained for the spheres. The balance between the shortwave forcing and longwave forcing is highly sensitive with respect to the assumed crystal shape and may even change the sign of the net forcing. The optical depth at which the mean diurnal radiative forcing changes sign from positive to negative varies from 4.5 to 10 for a surface albedo of 0.2 and from 2 to 6.5 for a surface albedo of 0.05 . Contrails are probably never that optically thick (except for some aged contrail cirrus), however, and so will not have a cooling effect on climate.
\end{abstract}

\section{Introduction}

Aviation influences the earth's atmosphere through the emission of gases and particles, which in turn affect climate both directly and indirectly. Water vapor and aerosol particles acting as cloud condensation nuclei are of special interest because they support cloud formation and modify cloud properties. Water vapor emitted by aircraft perturbs the background humidity in the atmosphere and can lead to the formation of contrails (lineshaped contrail) or "aviation induced cloudiness" (Sausen et al. 2005). The climate effect in terms of the radiative

Corresponding author address: Krzysztof M. Markowicz, Institute of Geophysics, Faculty of Physics, University of Warsaw, Pasteura 7, 02093 Warsaw, Poland.

E-mail: kmark@igf.fuw.edu.pl forcing of contrail clouds is highly uncertain because of a limited number of observed statistics of contrail parameters (e.g., Palikonda et al. 2005) and difficulties with the parameterization of contrails in general circulation models (Ponater et al. 2002; Rap et al. 2010; Kärcher et al. (2010). The Intergovernmental Panel on Climate Change (IPCC) "Aviation and Global Atmosphere" special report (Penner et al. 1999) provided an estimate for the radiative forcing of linear contrails that was in the range of $0.02 \mathrm{~W} \mathrm{~m}^{-2}$ for 1992, amounting to $40 \%$ of the total radiative forcing from aviation impact. The IPCC emphasized that the radiative forcing due to line-shaped contrails was known sufficiently to allow it to be attributed at least a "fair" level of scientific understanding. The "TRADEOFF" research project in 2005 led to updates in the IPCC's values (at the time of writing, a description of the study and its objectives 
could be found online at http://www.iac.ethz.ch/tradeoff/ srchidadb.html). The new studies gave $0.01 \mathrm{~W} \mathrm{~m}^{-2}$ as the best estimate of the mean global contrail radiative forcing in 2000 (Sausen et al. 2005). The last IPCC report (Solomon et al. 2007) maintained $0.01 \mathrm{~W} \mathrm{~m}^{-2}$ for the global mean forcing but attributed to it a "low" level of scientific understanding.

The relatively small values of the global net contrail radiative forcing arise as a residual from subtracting two comparatively larger numbers of a negative shortwave (SW) and positive longwave (LW) radiative forcing (Marquart et al. 2003; Rap et al. 2010; Stuber et al. 2006; Myhre and Stordal 2001; Meerkötter et al. 1999). The magnitudes of both the SW and LW effects, and thus the magnitude and sign of the net forcing, have been shown to depend on a number of factors. The investigated parameters include contrail optical depth (Kärcher et al. 2009; Meerkötter et al. 1999; Meyer et al. 2002; Frömming et al. 2011), contrail fraction (Rädel and Shine 2008; Marquart et al. 2003), surface albedo (Myhre and Stordal 2001; Meerkötter et al. 1999), and background cloud properties and contrail altitude (Rap et al. 2010; Rädel and Shine 2008; Myhre and Stordal 2001; Minnis et al. 1999). Uncertainties in the ice-crystal single-scattering properties (Yang et al. 2010; Kahnert et al. 2008) and the choice of radiative transfer models (Myhre et al. 2009) were also shown to influence the simulated contrail radiative forcing. Myhre et al. (2009) found large differences in the net forcing simulated with various radiative transfer codes. In addition, at high solar zenith angles the net forcings become very low and for some of the codes even change the sign.

In general, the LW warming is not expected to be balanced by the SW cooling, mainly because contrail optical depths are too small. Fu and Liou (1993) report that the net radiative forcing of cirrus clouds changes sign and becomes negative for clouds that have optical depths that are larger than 10. For comparison, contrailinduced cirrus clouds usually have optical depths that are below 2 (Haywood et al. 2009). Despite that fact, under certain conditions contrails might have a negative radiative forcing, as for instance was reported by Fortuin et al. (1995) and Rap et al. (2010). Rap et al. (2010) reported their negative values for daytime only and for latitudes between $45^{\circ}$ and $60^{\circ} \mathrm{N}$, whereas Fortuin et al. (1995) assumed very high contrail optical depths. These uncertainties in our understanding of the contrail radiative effect were one of the main motivations for performing this study: we investigate the conditions for which the contrail SW and LW radiative forcings balance each other and the net forcing becomes negative. In addition, it is unclear for which scenarios and contrail optical depths the positive forcing is most likely to reach its maximum. Previous studies reported a maximum of the net radiative forcing for optical depths between 2 and 3 (Jensen et al. 1994; Meerkötter et al. 1999) and of nearly 3 (Fu and Liou 1993). These values suggest that some observed contrail-induced cirrus clouds may already exert the strongest possible warming. Here we address some of the existing uncertainties by performing a detailed sensitivity study of contrail radiative-forcing computations on various parameters, primarily on the parameterization of ice-crystal shape and optical properties.

Radiative properties of ice particles have been studied before from various perspectives (Yang et al. 2010). In the past 10 years, numerous techniques were developed to calculate optical properties of particles with various morphologies. The microphysical and optical properties of contrail and contrail-induced cirrus clouds are very different from those of natural cirrus clouds (Schröder et al. 2000; Kärcher et al. 2007). Unlike a natural cirrus cloud, which is characterized by a wide size spectrum of ice crystals, the contrail usually has a higher number concentration of small ice crystals (Heymsfield et al. 2010). In addition, contrail particles can contain sootthat is, black carbon, which further modifies their singlescattering properties (Hong et al. 2008).

A major problem in the modeling of optical properties of contrails is the nonsphericity of the ice crystals that form these clouds. This situation prevents efficient analytical treatment of their single-scattering properties. Most ice particles are considerably larger than the wavelengths of the incoming solar radiation, however. Therefore, the geometric-optics approximation offers a conceptually simple way to simulate scattering of SW radiation for almost arbitrarily shaped objects. Ice particles are unfortunately too small to apply geometric methods in the LW range. The LW single-scattering parameters are typically computed using various methods such as the finite-difference time-domain technique (Sun et al. 1999; Yang and Liou 1996), the T-matrix method (Mishchenko and Travis 1994), the discrete dipole approximation (DDA; Draine and Flatau 1994), and the Lorenz-Mie theory (Bohren and Huffmann 1983) for equivalent spheres (Yang et al. 2005). In general, computations of optical properties for more complicated particle habits are very difficult and are computationally inefficient. This is because scattering properties, such as the scattering and absorption cross section, phase function, asymmetry parameter, and backscattering cross section need to be evaluated for a large number of cases, including various particle sizes, shapes, and random orientations against the incoming radiation. Some commonly used databases of optical properties include those reported by Yang et al. (2005) for the nearto far-infrared regimes and Yang et al. (2000) for the 
TABLE 1. Description of contrail-cloud optical models used in this study.

\begin{tabular}{|c|c|c|c|}
\hline & Optical model & Shape & Model description \\
\hline 1 & Flat optics & Not defined & $\begin{array}{l}\text { Single-scattering albedo } 1 \text { for SW and } 0.6 \text { for } \mathrm{LW} \text {, } \\
\text { asymmetry parameter } 0.8 \text {. }\end{array}$ \\
\hline 2 & Strauss contrails & Mixture & $\begin{array}{l}\text { Based on geometric optics in LW (hexagonal shapes) } \\
\text { and Mie calculations in SW (spheres). }\end{array}$ \\
\hline 3 & Droxtal & Droxtals & Optical particles based on improved geometrical-optics \\
\hline 4 & Hex pla & Hexagonal plates & method (Yang et al. 2005) in SW and finite-difference \\
\hline 5 & Hex col & Hexagonal columns & $\begin{array}{l}\text { time domain (Yang et al. 2000), the T matrix (Mishchenko } \\
\text { and Travis 1994) in LW. The particle's aspect ratio } \\
\text { depends on the particle size. }\end{array}$ \\
\hline 6 & DDA plates 0.2 & Hexagonal plates with aspect ratio 0.2 & Optical properties based on DDA (Draine and Flatau 1994) \\
\hline 7 & DDA plates 0.5 & Hexagonal plates with aspect ratio 0.5 & in LW and geometric method (Macke and Mishchenko \\
\hline 8 & DDA hex 3 & Hexagonal columns with aspect ratio 3 & 1996) in SW. \\
\hline 9 & DDA hex 5 & Hexagonal columns with aspect ratio 5 & \\
\hline 10 & Mie spherical & Homogenous spherical particles & Lorenz-Mie theory \\
\hline
\end{tabular}

visible- to near-infrared spectral regions. The DDA technique has been successfully used by Liu (2008) in the microwave range for 11 particle shapes. Other studies and databases on ice and snow scattering properties in the microwave range using alternative computational methods can be found in Evans and Stephens (1995), Kim (2006), and Hong (2007).

In this paper we investigate the influence of various ice-crystal shape parameterizations on the contrail SW and LW radiative forcings. Our major focus is to investigate conditions for which the contrail SW and LW radiative forcings balance each other and the net radiative forcing changes sign from positive to negative. In section 2 we describe the methods used to derive icecrystal optical properties and to perform radiative-forcing calculations. Section 3 compares optical properties such as the single-scattering albedo (SSA) and asymmetry parameter obtained from different computational techniques and databases. In section 4 , radiative forcing for different contrail models is studied and the results of various sensitivity studies are presented. Section 5 summarizes the results.

\section{Methods}

\section{a. Contrail-cloud models}

Contrail clouds consist of ice crystals having a variety of shapes, sizes, and volumes. Moreover, as a contrail evolves in time, spreads, and condenses more water vapor from the ambient air, the ice-crystal concentration and geometry may also undergo thorough changes. Some in situ observations of contrail ice particles indicate that crystal habits are dominated by the hexagonal columns and plates. Goodman et al. (1998) found, on the basis of measurements, that the average aspect ratio of hexagonal columns was less than 2. Because of a limited number of such observations, however, the average aspect ratios of contrail particles and their temporal evolution are still poorly understood.

To effectively assess contrail radiative effects, certain simplifications regarding the shape and size distribution of particles forming these clouds have to be made because our knowledge about contrail particle shapes and size distribution is still incomplete. For such simplified contrailcloud models, optical databases have to be established prior to radiative-forcing calculations. Here we describe in detail a method that was used to obtain optical databases for 10 various contrail-cloud models, listed in Table 1. Four of these models (models 6, 7, 8, and 9) required numerically expensive single-scattering derivations for individual ice crystals. Another four (models $2,3,4$, and 5) are based on optical databases found in the literature, and the remaining two (models 1 and 10) include highly simplified models, used here for reference.

\section{b. Hexagonal columns and plates (models 6-9 in Table 1)}

Four new crystal shape models have been developed in this study in an attempt to achieve the most complete view on the range of results provided by various contrail parameterization approaches. The new shapes include hexagonal plates with aspect ratios of 0.2 and 0.5 as well as hexagonal columns with aspect ratios of 3 and 5 . Single-scattering properties in the SW and LW electromagnetic spectra are derived for these shapes, for particle maximum dimensions ranging from 2 to $200 \mu \mathrm{m}$. The choice of wavelengths used to characterize the spectral dependence of single-scattering properties was based on the imaginary part of the refractive index of ice (see Fig. 1). The spectral points were selected manually to reflect variability of this parameter; therefore, the chosen wavelengths are located close to local maxima or 


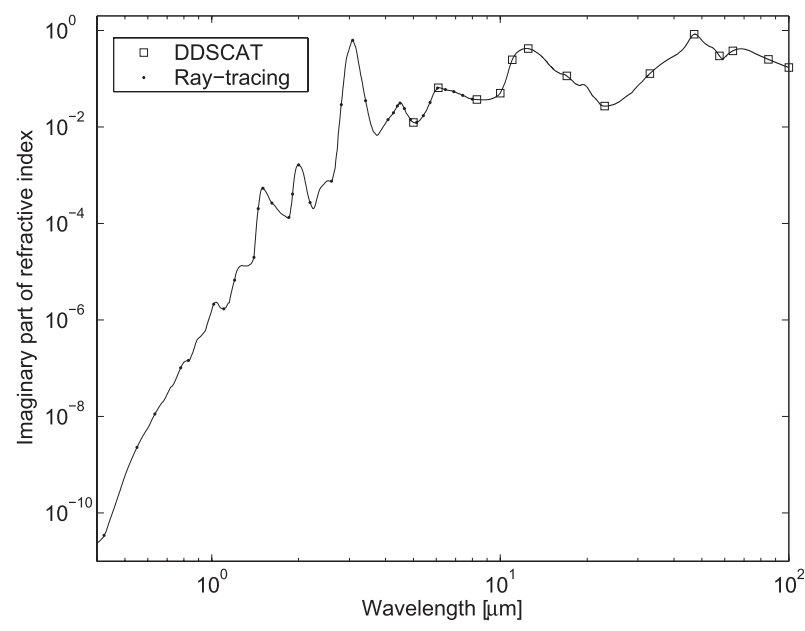

FIG. 1. Imaginary part of the refractive index of ice as a function of wavelength. Dots and squares represent wavelengths used for single-scattering computations. The DDA method was used in the longwave regime, and the geometrical-optics method was used in the shortwave regime (for further description see the text).

minima of the refractive index. The number of selected points reflects a compromise between the computational time required to derive single-scattering properties and the fidelity with which the spectral variability is represented. Two computational methods suitable for the separate spectral regimes were applied: the discrete dipole approximation and the geometrical-optics approximation.

The DDA, a flexible method that accurately computes the radiation scattering by arbitrarily shaped and inhomogeneous particles (Draine and Flatau 1994, 2003), is used to compute single-scattering properties for nonspherical ice crystals. In the DDA technique an arbitrarily shaped target is represented by an array of polarizable point dipoles. The point dipoles occupy the position on a cubic lattice with a lattice spacing that is required to be small in comparison with any structural length in the target and the incident wavelength. To compute the scattering of electromagnetic waves by the target accurately, a large number of dipoles is typically required, which makes the method computationally expensive. Draine and Flatau $(1994,2003)$ show that the DDA method gives adequately accurate results (errors in the SSA are less than $1 \%$ ) if $|m| k d<1$, where $m$ is the refraction index, $d$ is the lattice space, and $k$ is the angular wavenumber. To compute the scattering phase function, a more stringent criterion of $|m| k d<0.5$ is required. We applied this criterion because the phase function is used to determine the asymmetry parameter.

In this study, randomly oriented ice crystals are considered. The orientation averaging of a quantity $\langle Q\rangle$ is obtained by (Draine and Flatau 2003)

$$
\langle Q\rangle=\frac{1}{8 \pi^{2}} \int_{0}^{2 \pi} d \beta \int_{-1}^{1} d \cos \theta \int_{0}^{2 \pi} d \phi Q(\beta, \theta, \phi),
$$

where $\beta, \theta$, and $\phi$ are the three angles defining the orientation of the ice crystal relative to the incoming radiation. We used a number of 10,12 , and 20 angles for $\beta$, $\theta$, and $\phi$, respectively, to average over the whole range of orientations. Sensitivity tests with a larger number of angles (not shown here) proved that this configuration is sufficient to accurately represent single-scattering properties of randomly oriented particles. A larger number of orientation angles slows down the computations substantially, effectively reducing the range of particle sizes to be covered by DDA. Moreover, the DDA method is not computationally efficient for particles with a size parameter that is larger than 20-30. Therefore, we used this technique in the longwave radiation regime only, with the smallest wavelength set to $5 \mu \mathrm{m}$ (see Fig. 2). Version 6.1 of the discrete dipole approximation for scattering and absorption of light by irregular particles (DDSCAT) computer code (Draine and Flatau 2003) was used for the computations.

To calculate the single-scattering properties at the SW spectrum we used the geometrical-optics approximation. This method offers a conceptually simple although time-consuming way to simulate optical properties of almost arbitrarily shaped targets. The geometric approach is based on the assumption that the light may be thought of as consisting of separate localized rays that travel along straight-line paths. The ray-tracing technique (Rockwitz 1989; Macke et al. 1996; Macke and Mishchenko 1996) includes rays externally refracted by the particle and rays refracted into the particle where they could be absorbed or they may escape outside after possibly several internal reflections. Because in the geometric-optics technique one-half of the scattered radiation is due to diffraction that does not absorb light, this method cannot be used at wavelengths with significant absorption. In the SW radiation range the imaginary part of the refractive index of ice is fortunately small $(<0.01)$, with the exception of the absorption band around $3.1 \mu \mathrm{m}$. Comparison of the SSA (for spherical particles) derived by the ray-tracing and Lorenz-Mie methods shows that in the spectral band of 2.85-3.2 $\mu \mathrm{m}$ the geometric technique overestimated SSA by up to $10 \%$. The impact of these differences on the radiativeforcing simulations is marginal, however, because only about $0.65 \%$ of the solar flux is located in this spectral band. Macke and Mishchenko (1996) show that, for large spherical particles with a size parameter $(2 \pi r / \lambda$, where $r$ is the radius and $\lambda$ is the wavelength) of about 1000 and at nonabsorbing wavelengths, the scattering phase function derived using the ray-tracing method and 


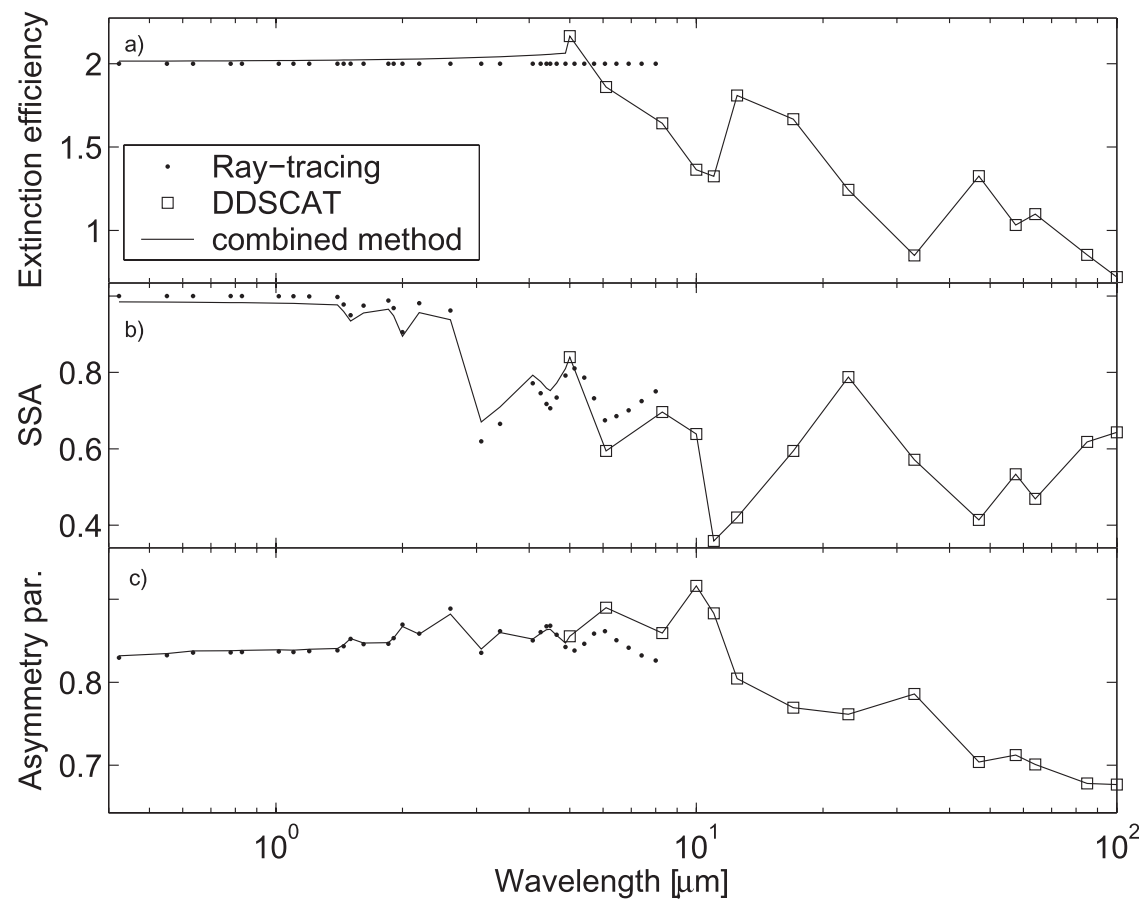

FIG. 2. Comparison of the (a) extinction efficiency, (b) single-scattering albedo, and (c) asymmetry parameter obtained from DDSCAT (open squares) and the ray-tracing method (dots) for randomly oriented hexagonal column crystals with an aspect ratio of 3 . The solid line shows combined optical properties from both techniques.

the Lorenz-Mie theory agree remarkably well. Furthermore, using the geometric-optics and T-matrix (Mishchenko and Travis 1994) methods for moderately absorbing spheroid and cylinder particles, they found an excellent agreement for size parameters of about 60. In the study presented here, the configuration of the raytracing simulations is the following: 200 rays per one crystal orientation, 2000 crystal orientations, and a maximum of 50 internal reflections. Other studies suggest that a more generous setting should be used to achieve stable and accurate results [e.g., Macke et al. (1996): 300 rays per orientation and 30000 orientations). Our sensitivity tests indicate that the optical properties differ by less than $0.1 \%$ between the two configurations, which justifies the use of the less computationally expensive settings.

\section{c. Other models (models 1-5 and 10 in Table 1)}

Parameterization approaches for cirrus-cloud properties (including contrails) adopted from existing literature are 1) the scattering and absorption property database for nonspherical ice particles in the visible and near-infrared (Yang et al. 2000) and far-infrared (Yang et al. 2005) spectral regions and 2) the optical database from Strauss et al. (1997). Yang et al. (2000, 2005) defined singlescattering properties of ice crystals randomly oriented in space and having the following shapes: hexagonal plates, hexagonal columns, and droxtals. For these shapes the particles' aspect ratio depends nonlinearly on the particles' size. The aspect ratios averaged over the size distribution are about 1.43 for hexagonal columns and 0.59 for hexagonal plates. For SW radiation, the singlescattering properties were computed based on the improved geometrical-optics method (Yang et al. 2000). For the middle- and far-infrared spectral range the authors used a combination of the finite-difference time-domain (Yang and Liou 1996; Sun et al. 1999), T-matrix (Mishchenko and Travis 1994), and improved geometrical-optics methods and the Lorenz-Mie theory. The Strauss contrail model (Strauss et al. 1997) assumes a mixture of hexagonally shaped particles. The optical properties are derived using the geometric-optics method in the SW range and the Lorenz-Mie theory (for volume equivalent spheres) in the LW regime.

Apart from the models that are based on realistic crystal shapes we used two idealized cirrus-cloud models: the flat-optics model and the spherical-particles model. The first model (flat optics) is characterized by the wavelength- and shape-independent optical properties. The SSA is set to 1 and 0.6 in the SW and LW regimes, respectively. The asymmetry parameter is set to 0.8 (Myhre et al. 2009). The second model (Mie spherical) is derived from the Lorenz-Mie theory for 
homogenously spherical particles (Heymsfield and Platt 1984). Both models, despite being simplified versions of the real contrail clouds, are useful reference points for more sophisticated and observationally justified models.

\section{d. Contrail crystal size distribution}

For all contrail-cloud models, wavelength-dependent optical properties such as the extinction coefficient, SSA, phase function, and asymmetry parameter were derived. Results were averaged over a size spectrum of contrail particles. The size distribution of different ice habits is described in terms of the maximum size $L$, which is defined as the longest dimension of the ice crystal. Measurements of contrail particles with impactors and optic probes reveal a large variety of size distributions. The results depend primarily on cloud age, ambient humidity, and ambient aerosol. In this study, the particle size distribution is taken from Strauss et al. (1997) and corresponds to a contrail age from about $10 \mathrm{~min}$ to $1 \mathrm{~h}$. This function covers particles with $L$ varying from 2 to $200 \mu \mathrm{m}$. For particles that are smaller than $20 \mu \mathrm{m}$, the size distribution is based on measurements. For crystals that are larger than $20 \mu \mathrm{m}$, the size distribution is parameterized according to Heymsfield and Platt (1984), with the amendments by Liou (1992). The Strauss et al. (1997) size distribution is defined for the following five bin intervals: 2-6, 6-20, 20-40, 40-90, and 90-200 $\mu \mathrm{m}$, with the particle concentrations of $2.91 \times$ $10^{5}, 6.67 \times 10^{5}, 3.05 \times 10^{4}, 5.51 \times 10^{3}$, and $2.42 \times$ $10^{3} \mathrm{~m}^{-3}$, respectively. This corresponds to an overall particle number concentration of $1.0 \mathrm{~cm}^{-3}$ and an ice water content of $2.08 \times 10^{3} \mathrm{~g} \mathrm{~m}^{-3}$.

Applying the same particle size distribution to various ice-crystal models results in slightly different effective radii characterizing these models. Also, optical properties have different wavelength dependence and the optical thickness varies between models. For radiativeforcing calculations, however, the optical thickness of all crystal models is scaled to the same value at a $550-\mathrm{nm}$ wavelength. Such an approach preserves variability in the shapes of wavelength-dependent optical parameters and at the same time facilitates comparison among various contrail models.

\section{e. Radiative transfer model}

The contrail radiative forcing is a perturbation of the earth-atmosphere system net radiative fluxes caused by the contrails. It is defined as the difference between the net (down minus up) radiative flux for the atmosphere with contrails and the net radiative flux without contrails:

$$
\mathrm{FOR}=\left(F_{\mathrm{con}}^{\downarrow}-F_{\mathrm{con}}^{\uparrow}\right)-\left(F_{\mathrm{clr}}^{\downarrow}-F_{\mathrm{clr}}^{\uparrow}\right),
$$

where $F_{\text {con }}^{\uparrow}\left(F_{\text {con }}^{\downarrow}\right)$ and $F_{\text {cle }}^{\uparrow}\left(F_{\text {cle }}^{\downarrow}\right)$ indicate the upward (downward) contrail-modified and contrail-free solar plus infrared fluxes. In this study we discuss instantaneous radiative forcings from contrails [Eq. (2)] defined at the top of the atmosphere (TOA) for a fixed set of ambient parameters, and diurnal mean radiative forcings averaged over $24 \mathrm{~h}$ with a 15 -min time interval. The term instantaneous radiative forcing has usually been used as a distinction from the stratosphere-adjusted forcing as used by the IPCC (e.g., Solomon et al. 2007) but this is not an issue here, because stratospheric temperature adjustment in not included in our radiative-forcing calculations. Further, the atmosphere is assumed to be free from any background aerosols and clouds.

The moderate-resolution atmospheric radiance and transmittance model (MODTRAN), version 4.1, (Berk et al. 1998) radiative transfer code with the discrete ordinate radiative transfer model (DISORT; Stamnes et al. 1988) solver was used for the radiative transfer calculations. The DISORT model includes multiple-scattering effects. Eight streams and the MODTRAN band model "M" (17 absorption coefficient and $17 k$ values) for the SW and K correlated (33 $k$ values) for the LW were used in the simulations. A molecular spectroscopy database with a resolution of $1 \mathrm{~cm}^{-1}$ in the $S W$ and $15 \mathrm{~cm}^{-1}$ in the LW was used. MODTRAN errors are about $2 \%$ for the aerosol-free solar fluxes [cf. Intercomparison of Radiation Codes in Climate Models (Fouquart et al. 1991; Halthore et al. 2005)]. A good agreement in the radiative forcing computed by the MODTRAN model and a lineby-line code was found by Myhre et al. (2009).

\section{Contrail optical properties}

\section{a. Comparison of DDA and ray-tracing methods}

In this section single-scattering properties of ice particles obtained from the DDA and geometric ray-tracing methods are discussed. In general, the two methods are applicable to different spectral regimes, but an overlapping spectral range $(5-8 \mu \mathrm{m})$ can be established. This spectral range allows formulating a consistent optical database. Because of computational constraints it was not feasible to perform DDA calculations for wavelengths that are shorter than $5 \mu \mathrm{m}$ (the number of dipoles at $5 \mu \mathrm{m}$ already exceeds $10^{6}$ ).

Figures 2 and 3 present the single-scattering properties of randomly oriented hexagonal crystals with an aspect ratio of 3 (Fig. 2) and hexagonal plates with an aspect ratio of 0.2 (Fig. 3) obtained from the DDA method (open squares) and the ray-tracing method (solid dots). The extinction efficiency (Fig. 3a), the SSA (Fig. 3b), and the asymmetry parameter (Fig. 3c) were 


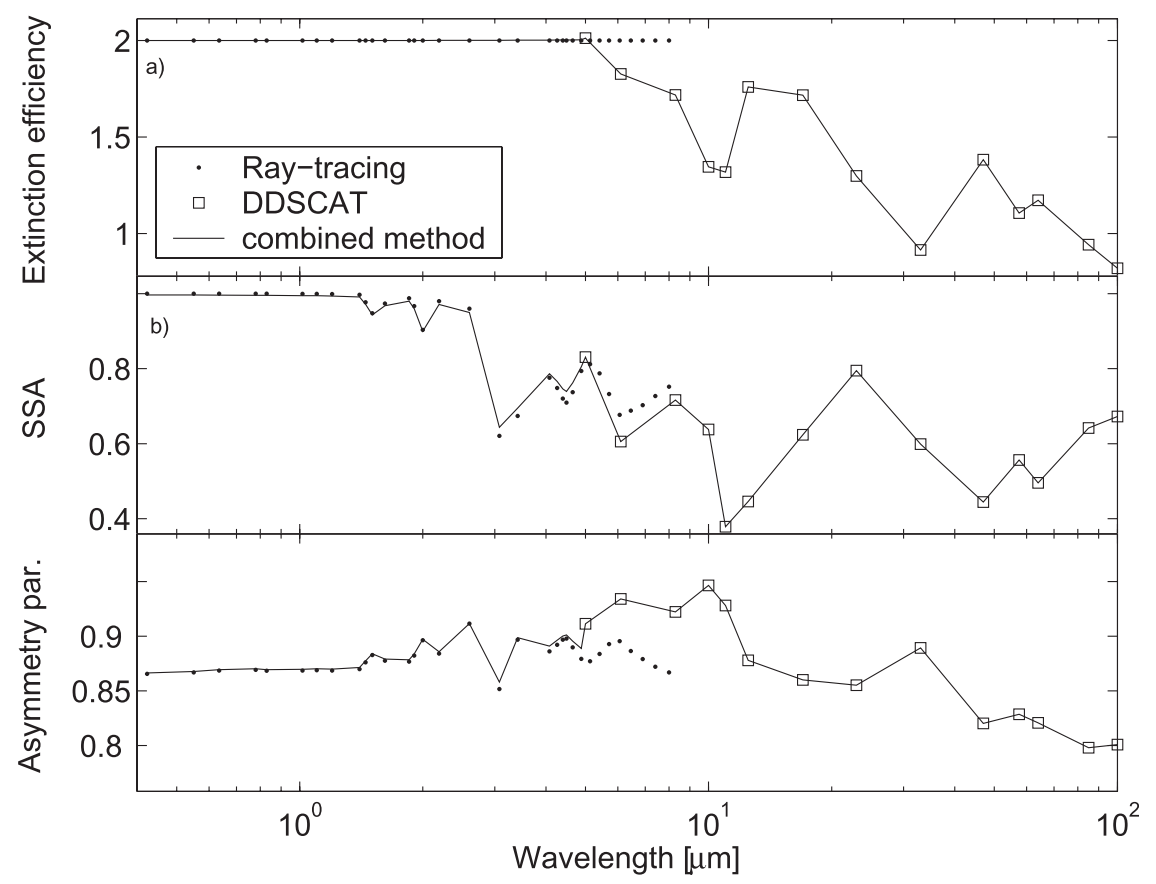

FIG. 3. As in Fig. 2, but for hexagonal plate crystals with an aspect ratio of 0.2.

averaged over the particles' size distribution taken from Strauss et al. (1997). The extinction efficiency in the raytracing method is always equal to 2 , which affects the accuracy of this method, especially for larger wavelengths (and smaller size parameters). Because of this fact, the results of the DDA calculations are considered to be more accurate in the transition regime and are given higher weighting during merging. The SSA and the asymmetry parameter show similar wavelength dependence in the overlapping zone. The differences between both methods can be up to $10 \%-15 \%$, however, and increase with wavelength.

To perform radiative-forcing calculations, the singlescattering properties in the whole solar and infrared spectra have to be defined. The single-scattering properties obtained from both methods are combined using a weighting function. In the transition region the DDA results are chosen as the most accurate. Toward shorter wavelengths the ray-tracing results are combined together with the DDA results from the 5- $\mu \mathrm{m}$ wavelength. The weight given to the DDA results is a function that decreases exponentially with the spectral distance from $5 \mu \mathrm{m}$. The combined optical properties are shown as solid lines in Figs. 2 and 3. Note that the existing differences between both methods are not very crucial because the radiative fluxes in the transition region are small. Any perturbation induced by contrails in this spectral range will therefore provide only a slight contribution to the integrated radiative forcing.

\section{b. Single-scattering properties of the ice-crystal models}

In this section optical properties of some of the contrailcloud models listed in Table 1 are briefly analyzed. Figure 4 shows the wavelength dependency of the SSA for seven ice-crystal models after averaging over the respective size distribution. We omitted some of the models [flat optics, DDA plates 0.5, and DDA hex (for hexagonal) 3] for a better clarity of the figures. An equivalent comparison but for the asymmetry parameter is presented in Fig. 5.

Figures 4 and 5 show that there can be substantial differences in optical properties among the models. These differences result mostly from distinct ice habits and various aspect ratios among the contrail models but also from different computational techniques. The differences in the SSA reach up to 0.1 in the infrared range (Fig. 4), whereas the SW values are very similar among models because of the small absorption in this range. The asymmetry parameter has larger variability, especially in the SW radiation regime (Fig. 5). The new models (denoted DDA hex col and DDA hex pla) with the fixed aspect ratios diverge from the previous estimates by Yang et al. $(2000,2005)$ and Strauss et al. (1997). The simulations presented here give values of asymmetry parameter in the SW that are more similar to the ones obtained for the spherical particles and are higher by around 0.1 when compared with the other 


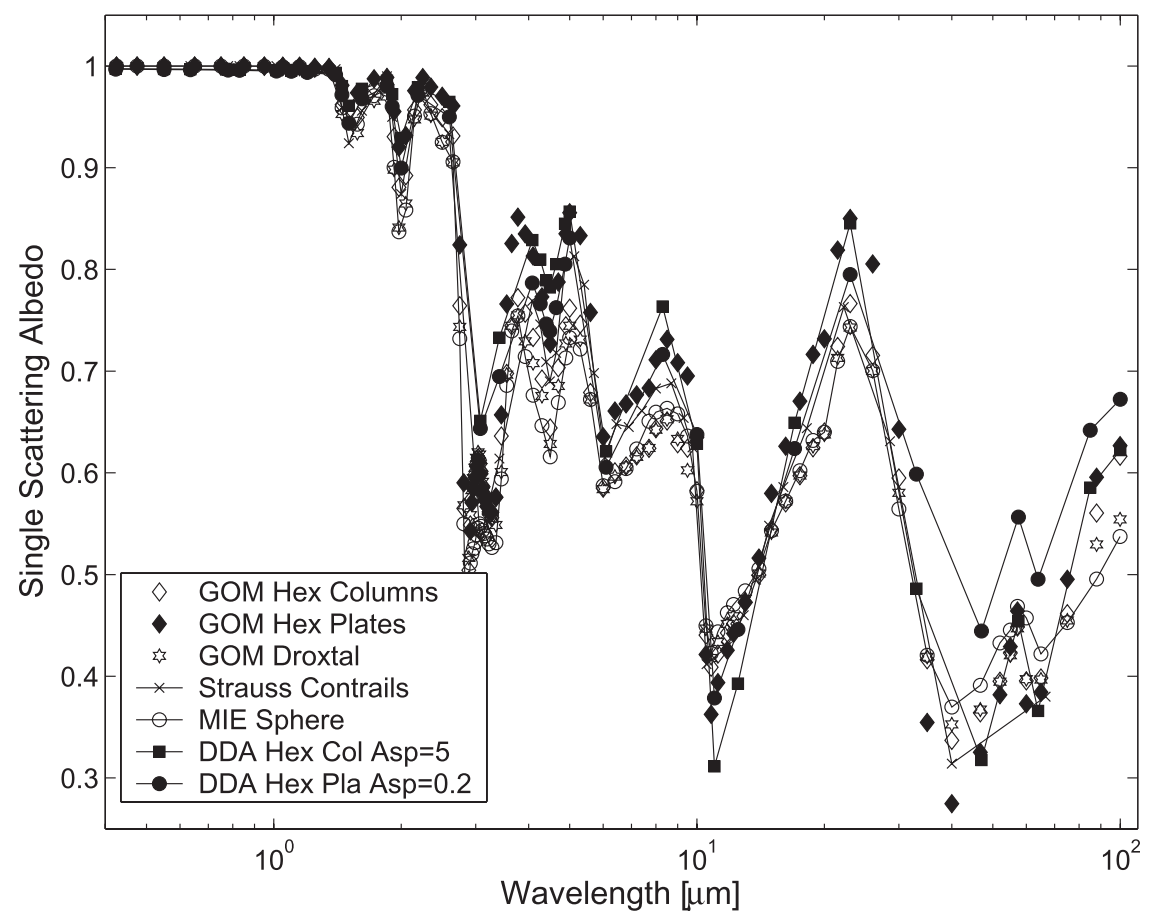

FIG. 4. Single-scattering albedo as a function of wavelength for several contrail-cloud models. The first three crystal types (open diamonds, solid diamonds, and hexagrams) are based on Yang et al. $(2000,2005)$ databases, the line with times signs shows contrails properties taken from Strauss et al. (1997), the line with open circles marks optical properties for spherical particle computed from the Lorenz-Mie theory, and the last two lines (with filled squares and filled circles) correspond to hexagonal columns with an aspect ratio of 5 and hexagonal plates with an aspect ratio of 0.2 (models 9 and 6 in Table 1). Optical properties were averaged over the ice-crystal size distribution given by Strauss et al. (1997).

models. Because of the high energy density in the SW range we expect the simulated differences to have substantial impact on radiative-forcing calculations. To shed some light on these large differences the asymmetry parameter dependence on aspect ratio at 550-nm wavelength is investigated. Randomly oriented hexagonal, cylindrical, and ellipsoidal particles averaged over the size distribution given by Strauss are considered. The results are shown in Fig. 6 . The asymmetry parameter has a strong dependence on the particles' habit and aspect ratio, reaching up to 0.1 within a single shape category. This dependence explains the variability observed in Fig. 5. Indeed, hexagonal particles with the most extreme aspect ratios ( 0.2 and 5$)$ have values closest to the Mie calculations, indicated in Fig. 6 by the solid circle. In addition, hexagonal particles with an aspect ratio near 1 have the lowest asymmetry parameter (14\% smaller than spherical crystals). This is in agreement with Fig. 5, where it can be observed that the Yang models have much smaller values than the Mie calculations-on average they have aspect ratios of 1.43 and 0.59 for hexagonal columns and plates, respectively. The ellipsoidal particles with an aspect ratio of 1 agree well with the Mie results, indicating a good accuracy of the ray-tracing method.

\section{Contrail radiative-forcing simulations}

As explained in section 2e, the MODTRAN version4.1 radiative transfer code is used for contrail forcing calculations. A reference configuration with a standard set of external parameters is defined. It is used for contrail model intercomparison and also as a reference point in various sensitivity test conducted in this study. In this reference configuration contrails are located between 10 and $11 \mathrm{~km}$ above the ground and have a fixed $1 \%$ cover fraction. Optical properties are assumed to be homogenous within the cloud layer. The contrail optical depth is set to 0.3 at $550-\mathrm{nm}$ wavelength and has a spectral dependence equivalent to the shape of the extinction coefficient of various contrail models. Additional specifications include a surface albedo of 0.2 , a surface emissivity of 1.0 , and atmospheric vertical profiles corresponding to the standard midlatitude summer. Unless stated otherwise, all further simulations 


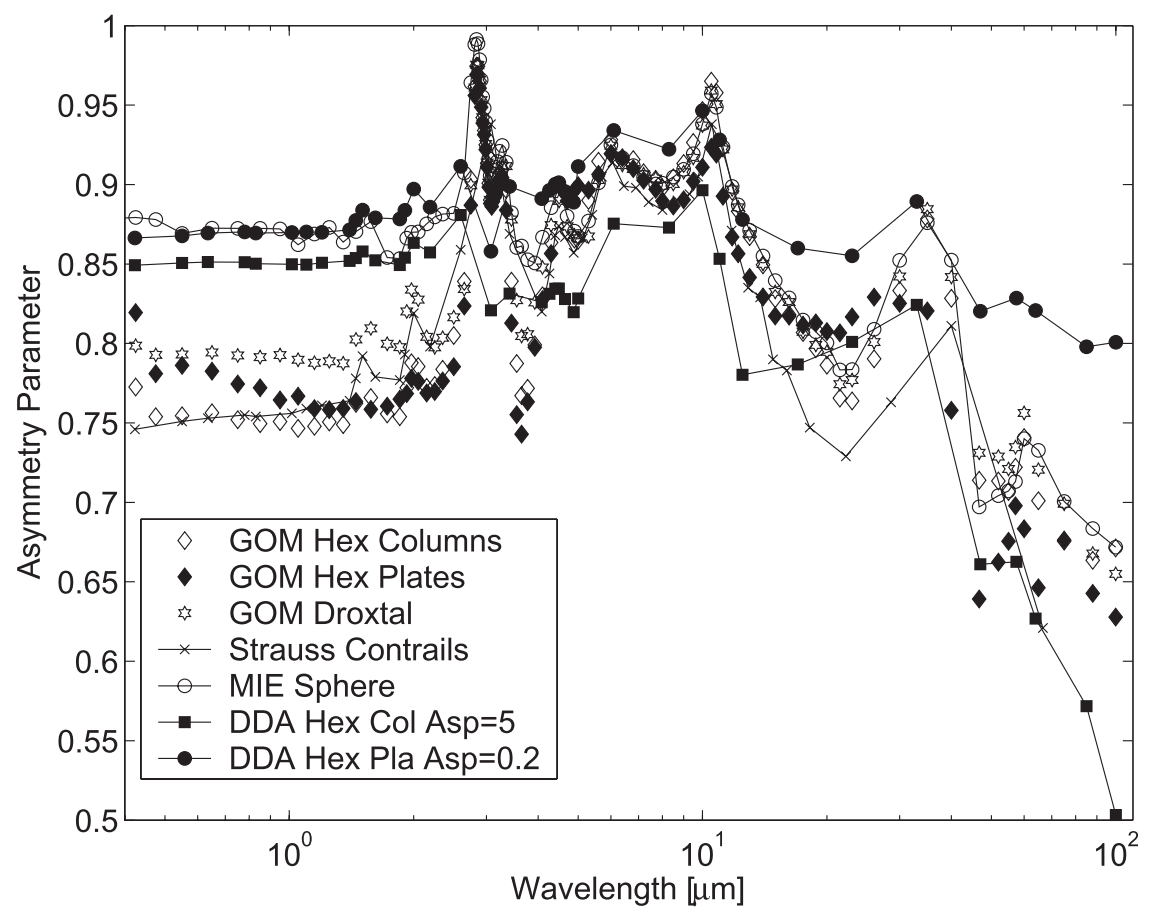

FIG. 5. As in Fig. 4, but for the asymmetry parameter.

and analyses were performed using this default contrail configuration.

\section{a. Comparison of instantaneous radiative forcing}

Figure 7 presents results from the simulations of instantaneous contrail radiative forcing at the TOA [Eq. (2)]. The simulations were performed with the reference contrail configuration and a solar zenith angle of $30^{\circ}$. There is a significant variation in the SW, LW, and net radiative forcings among different models. The solar radiative forcing is negative and varies between -0.15 and $-0.06 \mathrm{~W} \mathrm{~m}^{-2}$. This spread results mostly from the differences in the asymmetry parameter, because the SSA and the extinction coefficient are very similar in all contrail models. The smallest solar radiative forcing is simulated for the spherical particles, which have the largest asymmetry parameter (see Figs. 5 and 6). This is because a larger asymmetry parameter leads to less radiation scattered back to space.

The simulated infrared radiative forcing is positive and varies between 0.20 and $0.31 \mathrm{~W} \mathrm{~m}^{-2}$, indicating a distinctly stronger dependence of the longwave contrail forcing on crystal shape than has been reported in earlier studies (Meerkötter et al. 1999; Rap et al. 2010; Frömming et al. 2011). The lowest values are modeled for the most asymmetric cloud models-namely, the hexagonal plates and columns with aspect ratios of 0.2 and 5.0, respectively. This is probably due to the lower absorption resulting from the smaller volume of particles in these models. Unlike in the solar spectrum, the spread in the infrared forcing is influenced by all optical properties, the SSA, asymmetry parameter, and extinction

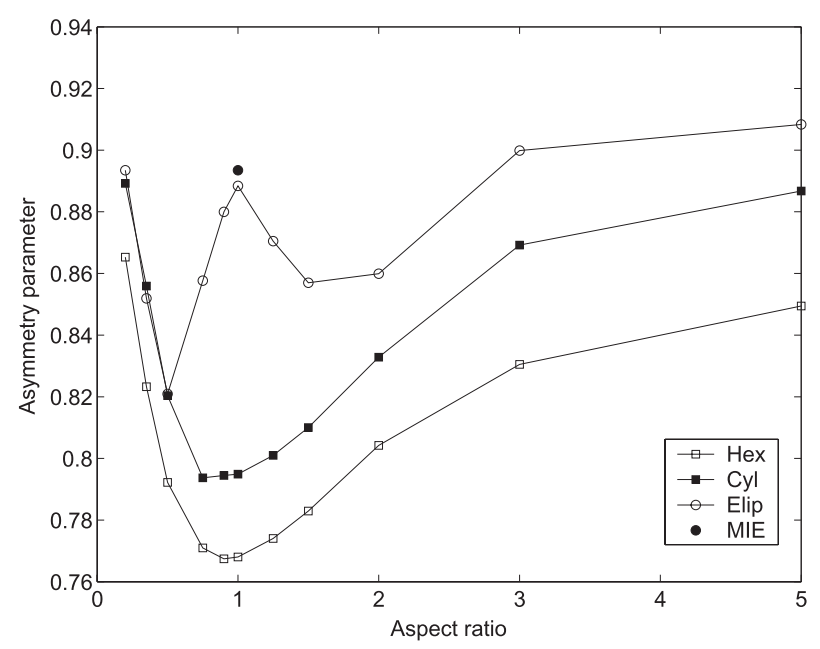

FIG. 6. Asymmetry parameter at $550 \mathrm{~nm}$ as a function of aspect ratio for randomly oriented hexagonal plates (open squares), cylinders (filled squares), and ellipsoids (open circles) obtained from the ray-tracing method. The dot shows the asymmetry parameter for spheres calculated from the Lorenz-Mie theory. The asymmetry parameters were averaged over the ice-crystal size distribution given by Strauss et al. (1997). 


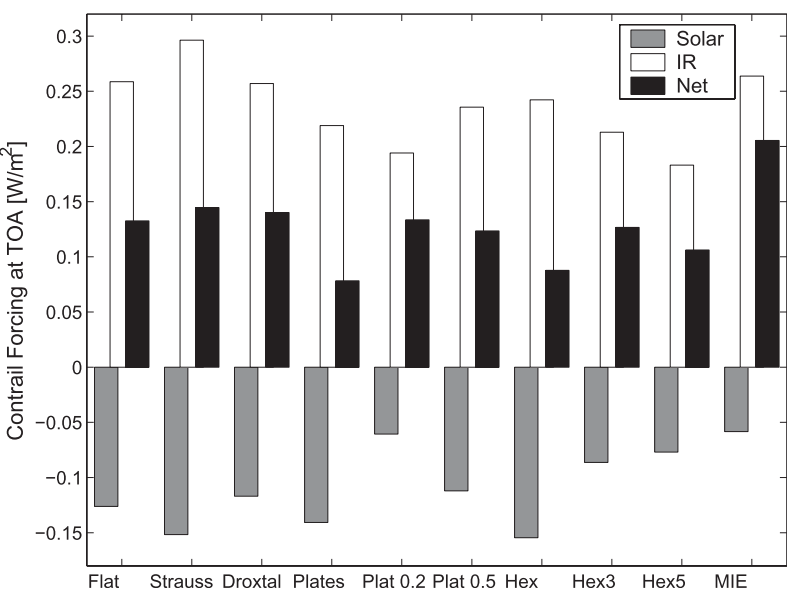

FIG. 7. Contrail solar (gray bars), infrared (white bars), and net (black bars) instantaneous radiative forcing at the TOA for various ice-crystal optical models. Simulations were performed with the default contrail configuration (see the text) and a solar zenith angle of $30^{\circ}$.

coefficient alike. Therefore it is difficult to describe the differences among models in terms of a single parameter. The combined net forcing is positive and ranges from 0.08 to $0.22 \mathrm{~W} \mathrm{~m}^{-2}$ depending on the contrail model. Even if we exclude the spherical particles the spread is still substantial and is equal to $0.08-0.16 \mathrm{~W} \mathrm{~m}^{-2}$. A factor-of-2 difference in the net forcing among nonspherical habits points to the need for a proper representation of ice shapes in atmospheric contrails.

Figure 8 shows the contrail SW, LW, and net radiative forcings for a solar zenith angle of $60^{\circ}$. In this case the SW and the $\mathrm{LW}$ radiative forcings are close to balance (note the difference in vertical scales between Figs. 7 and 8 ) and for most models lead to a negative net effect. The strongest cooling effect was simulated for the hexagonalcolumns and -plates contrail models taken from the Yang et al. $(2000,2005)$ databases. It is evident that the sign and magnitude of instantaneous net radiative forcing depend on the solar zenith angle. Other factors that can affect the net forcing include the contrail optical depth, surface albedo, and contrail altitude. We examine these dependencies in more detail.

Figure 9 shows the simulated TOA net radiative forcing as a function of contrail optical depth. The net radiative effect increases with optical depth, reaches a maximum at some optical depth, and after that gradually decreases and eventually becomes negative. The initial increase in warming is influenced by the infrared forcing, which intensifies as the optical depth becomes larger. At some point the solar cooling effect starts dominating over the infrared warming, however. This is because contrail clouds with optical thickness of about $2-5$ can be already considered to be a blackbody in the

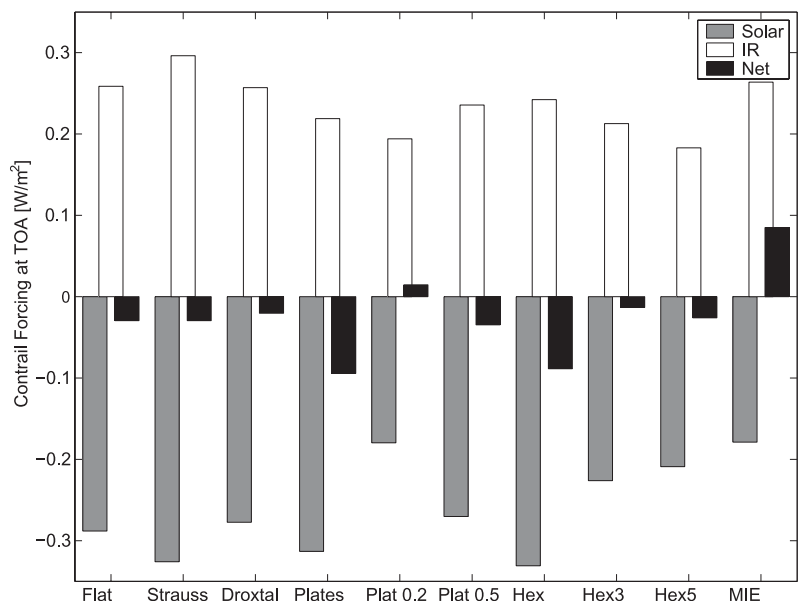

FIG. 8. As in Fig. 7, but for a solar zenith angle of $60^{\circ}$.

infrared, and a further increase of optical depth does not change the amount of radiation absorbed by these clouds. This saturation effect makes the infrared forcing highly nonlinear with respect to the optical depth (Jensen et al. 1994). As a consequence, the solar cooling becomes eventually stronger than the infrared warming and the simulated net forcing becomes negative. There is large variability among the contrail models in the maximum forcing magnitude and the critical optical depth (COD) at which the net effect changes sign. Figure 10 depicts these differences in a transparent way. For the 10 contrail models considered here, Fig. 10a shows the optical depths at which maximum forcing values are reached, Fig. 10b shows the magnitudes of these maximum forcings, and Fig. 10c presents the CODs at which the net forcing becomes negative. The maximum net forcing is usually achieved at optical depths that are higher than 0.5 , with only two optical models below this level (models 4 and 5 in Table 1). Given that observed contrail optical depths are generally smaller than 0.4 (Kärcher et al. 2009; Palikonda et al. 2005; Meyer et al. 2002), we can conclude that an instantaneous contrail radiative forcing at high solar elevations is mostly positive. The contrail-induced cirrus clouds are usually thicker than linear-shaped contrails, however. Haywood et al. (2009) analyzed satellite observations and showed that optical thicknesses of these clouds vary between 0.4 and 1.95. Thus, the contrail-induced cirrus may be optically thick enough to reach the maximum of the net radiative forcing. This maximum (see Fig. 10b) varies from $0.05 \mathrm{~W} \mathrm{~m}^{-2}$ for models 4 and 5 , through $0.18 \mathrm{~W} \mathrm{~m}^{-2}$ for the hexagonal plates with an aspect ratio of 0.2 (model 6), to $0.31 \mathrm{~W} \mathrm{~m}^{-2}$ for the spherical particles. As indicated in Fig. 10c, the simulated CODs are usually above 1.0, a value that is only seldom exceeded by observed contrails. As is evident from Fig. 8, the CODs will be 


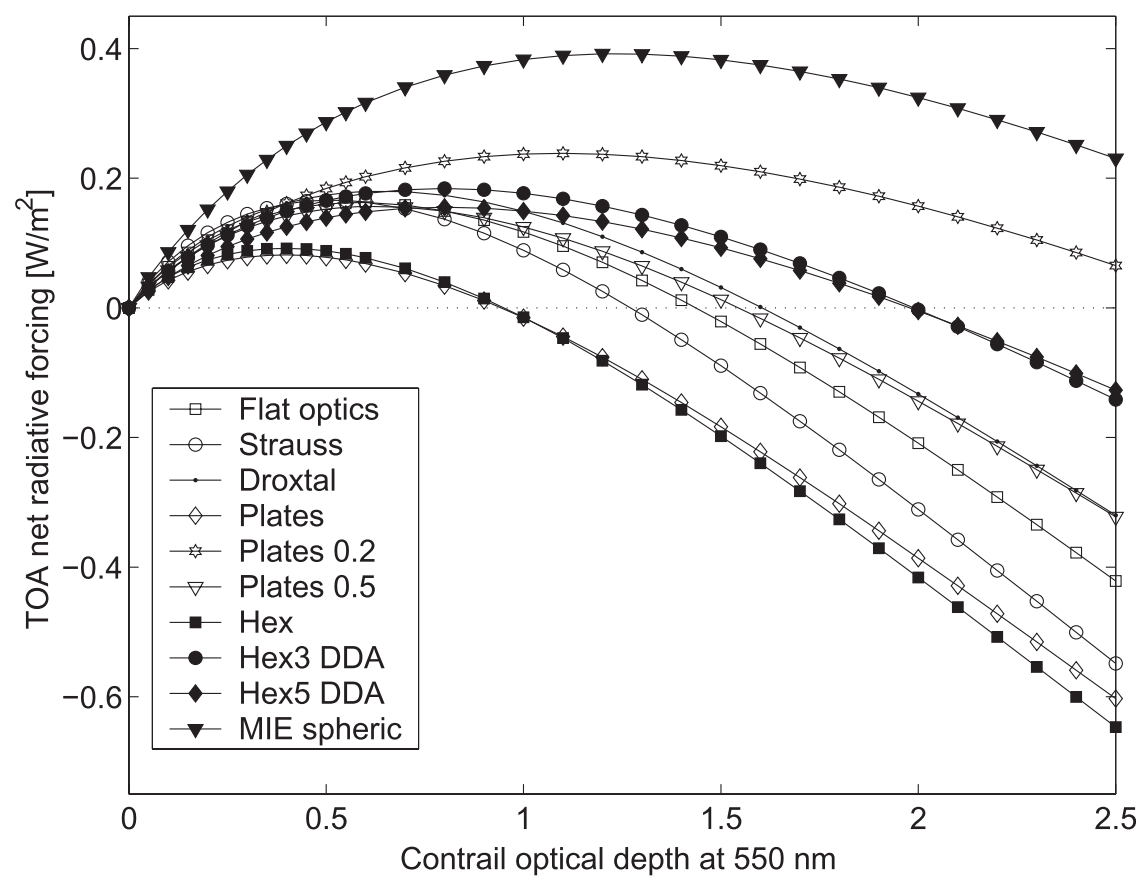

FIG. 9. Contrail instantaneous net radiative forcing at the TOA as a function of optical thickness at $550 \mathrm{~nm}$ for various ice-crystal optical models. Simulation was performed on the basis of the MODTRAN code with a solar zenith angle of $30^{\circ}$ and a surface albedo of 0.2 .

significantly lower for higher zenith angles, however. The surface albedo and contrail-top altitude are also factors that can affect those critical values.

Figure 11 examines the influence of contrail-top altitude (Fig. 11a), surface albedo (Fig. 11b), and solar zenith angle (Fig. 11c) on the optical depth at which the net forcing changes sign. The Strauss contrail model (model 2 ) is chosen to investigate these dependencies. Previous researchers (Penner et al. 1999; Rädel and Shine 2008) showed that the contrail forcing depends on the cloud's altitude. The results presented here (Fig. 11a) indicate that colder clouds have higher CODs, thus increasing the probability of a net warming effect. This is related to the temperature influence on the infrared forcing, because the solar component does not change with the cloud's temperature. The flattening above $13 \mathrm{~km}$ observed in Fig. 11a is a result of fairly constant temperatures in the assumed midlatitude summer profile. The four lines in Fig. 11a denote different surface albedo values. For small albedos and low contrail altitudes when the COD is zero, the instantaneous forcing will be negative regardless of the optical depth of the contrail. Higher albedo leads to larger COD values as the solar backscattering becomes less important over a brighter surface.

In a similar way, Fig. $11 \mathrm{~b}$ shows that for a given solar zenith angle and a contrail-top altitude of $11 \mathrm{~km}$ the COD increases with increasing albedo. Over dark surfaces the
CODs are relatively small because of the stronger SW cooling effects. Bright surfaces, on the other hand, increase the CODs significantly and make an instantaneous forcing always positive for contrails with realistic optical depths. Figure 11c investigates variations of CODs with the solar zenith angle. For a given surface albedo, the

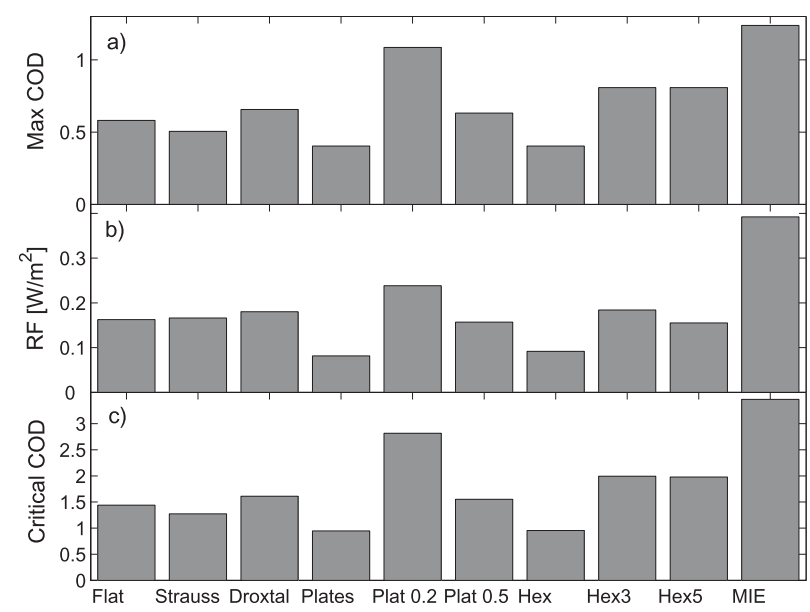

FIG. 10. For the 10 contrail optical models listed in Table 1: (a) contrail optical depth for which an instantaneous radiative forcing reaches maximum, (b) magnitude of the maximum forcing, and (c) the COD at which radiative forcing changes sign. Simulations were performed using the MODTRAN code with a solar zenith angle of $30^{\circ}$ and a surface albedo of 0.2 . 

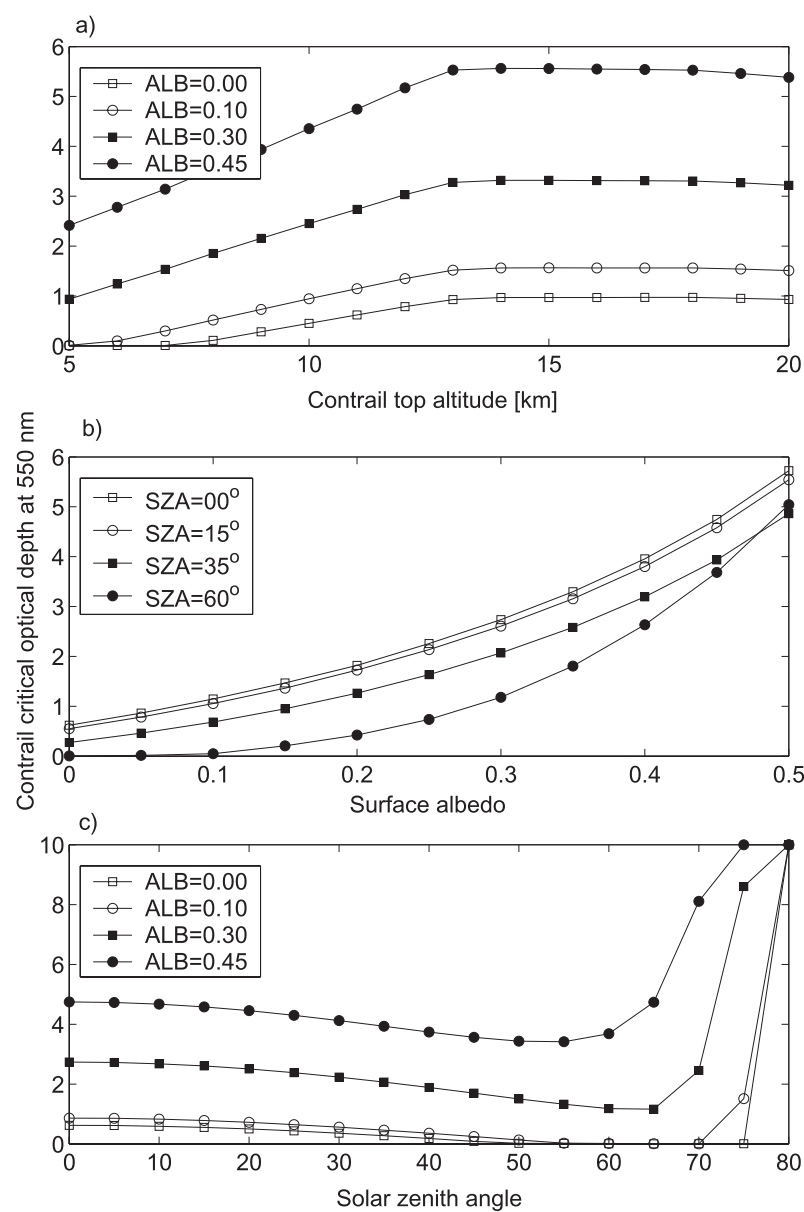

FIG. 11. Critical values of the contrail optical depth for which the TOA net radiative forcing is zero for different surface albedo, solar zenith angle, and contrail-top altitude: (a) COD as a function of the contrail-top altitude for different surface albedos and a solar zenith angle of $30^{\circ}$, (b) COD as a function of the surface albedo for different solar zenith angles, and (c) COD as a function of the solar zenith angle for different surface albedos. Computations were performed for the Strauss contrail optical model, and the contrailtop altitude is $11 \mathrm{~km}$ in (b) and (c).

COD decreases gradually as the zenith angle increases. The lowest values are reached at solar zenith angles between $50^{\circ}$ and $70^{\circ}$, depending on the surface albedo. Over dark surfaces the CODs drop to zero, meaning that at higher zenith angles the simulated net forcing is negative, even for contrails that are optically thin. The observed increase of COD values at low solar elevations is related to the reduction of SW radiative forcing (see section $4 b$ ).

\section{b. Diurnal variation of contrail radiative forcing}

Diurnal variability of an instantaneous contrail radiative forcing depends on several factors. Most important are parameters that affect the SW forcing component, like changes in the incoming solar radiation, scattering geometry, and surface albedo. Figure 12 presents the SW forcing as a function of the solar zenith angle for the contrail models listed in Table 1 . The SW forcing varies considerably even at a constant surface albedo and a constant optical depth. The amplitude between the lowest values at $0^{\circ}$ and the maximum forcings at around $70^{\circ}$ reaches about $0.2 \mathrm{~W} \mathrm{~m}^{-2}$ and depends slightly on the contrail model. The diurnal cycles obtained for each considered crystal model are generally similar. Significant differences occur both in the magnitude of the forcing maximum (between -0.25 and $-0.38 \mathrm{~W} \mathrm{~m}^{-2}$ ) and in the zenith angles for which these maximum values are reached (between $70^{\circ}$ and $75^{\circ}$ ), however. These differences result from the variations in asymmetry parameter among models, with a larger asymmetry parameter implying a smaller radiative impact induced by contrails at the TOA. Diurnal variability of the LW forcing is usually small and has a weak influence on the net forcing (Meerkötter et al. 1999; Dietmüller et al. 2008).

\section{c. Comparison of the mean diurnal radiative forcing for different ice-crystal habits}

In this section we discuss variability of the diurnal mean contrail radiative forcing with the particles' shape. Radiative transfer computations were performed with a 15-min time interval and averaged over $24 \mathrm{~h}$ for each of the contrail models listed in Table 1 . All input parameters in the simulations, except for the solar zenith angle, were kept constant. Diurnal cycle of the incoming solar radiation was set to be equivalent to conditions in June at $50^{\circ} \mathrm{N}$ latitude. Results of these calculations are presented in Fig. 13, which shows the same parameters as those in Fig. 10, but now for the mean diurnal radiative forcing. The lightly shaded bars represent the simulations performed with a surface albedo of 0.05 , and the dark bars correspond to a surface albedo of 0.2. The optical depths for which the net radiative forcing reaches maximum (Fig. 13a) are significantly larger than their instantaneous counterparts (Fig. 10a). This is related to the diurnal imbalance of LW and SW forcings, with the latter being zero during night time. The SW cooling effect is therefore reduced in comparison with the LW warming, shifting the maximum forcings toward larger optical depths. The simulated maximum net forcings (Fig. 13b) are also larger than the instantaneous ones (Fig. 10b) and range from 0.2 to $0.55 \mathrm{~W} \mathrm{~m}^{-2}$ for a surface albedo of 0.2 and from 0.08 to $0.4 \mathrm{~W} \mathrm{~m}^{-2}$ for an albedo of 0.05 . The CODs at which the net effect becomes negative are again higher for the daily-averaged forcings than for the instantaneous forcings (Fig. 13c). For a surface albedo of 0.05 , the CODs vary from 2 (hexagonal plates and hexagonal columns) to about 6 


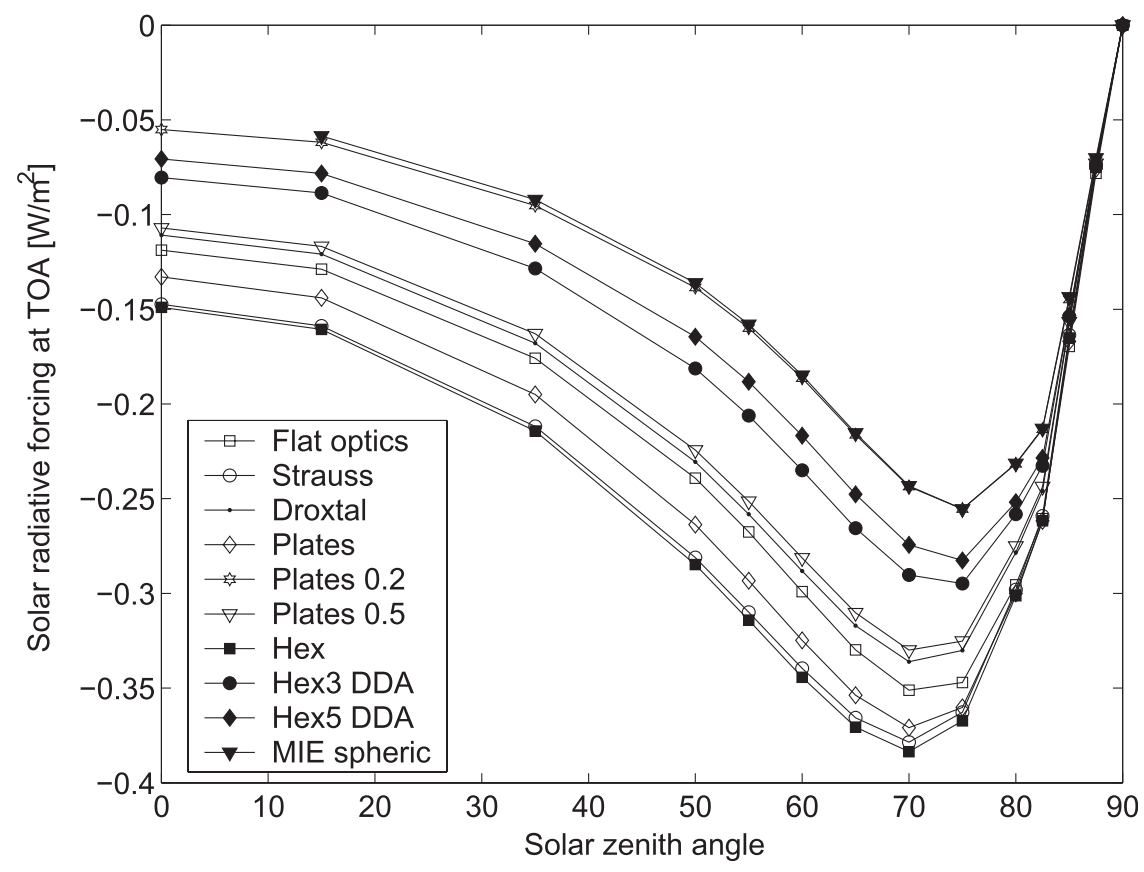

FIG. 12. Variability of the contrail solar radiative forcing with solar zenith angle for different crystal models.

(spherical particles). The CODs values are higher for a surface albedo of 0.2 and change from 4 to 10 . Such a large spread in the simulated CODs indicates substantial differences among the various contrail optical models. Even the lowest simulated COD values seem very large when compared with the observed contrail optical depths. This indicates that realistic contrails almost always have warming effect on climate and that the transition to cooling is unlikely even under a substantial increase in air-traffic-generated contrails. Contrailinduced cirrus clouds, which usually have higher optical depths, may approach these critical values, however (Fortuin et al. 1995). In addition, our results correspond to the summer conditions in midlatitudes only. Rap et al. (2010) pointed out that, although the daily averaged net contrail forcing is always positive, the daytime averaged values can be negative at latitudes between $45^{\circ}$ and $60^{\circ} \mathrm{N}$. This result is consistent with our simulations of the contrail radiative forcing at low solar elevations (see Fig. 8).

\section{Summary and conclusions}

In this paper, single-scattering properties of ice crystals and contrail radiative forcings are discussed. Optical properties in the SW and LW ranges were obtained by the ray-tracing and DDA methods, respectively. None of these techniques can be used for the whole radiative spectrum because of the physical and computational limitations. Good agreement of the SSA and the asymmetry parameter was obtained when using both methods in the transition spectrum range $(5-8 \mu \mathrm{m})$, however. Hence, a consistent optical database comprising both the solar and infrared spectral regimes was constructed. In this study, optical properties of the following randomly oriented crystal habits are directly computed: hexagonal plates with aspect ratios of 0.5 and 0.2 , hexagonal

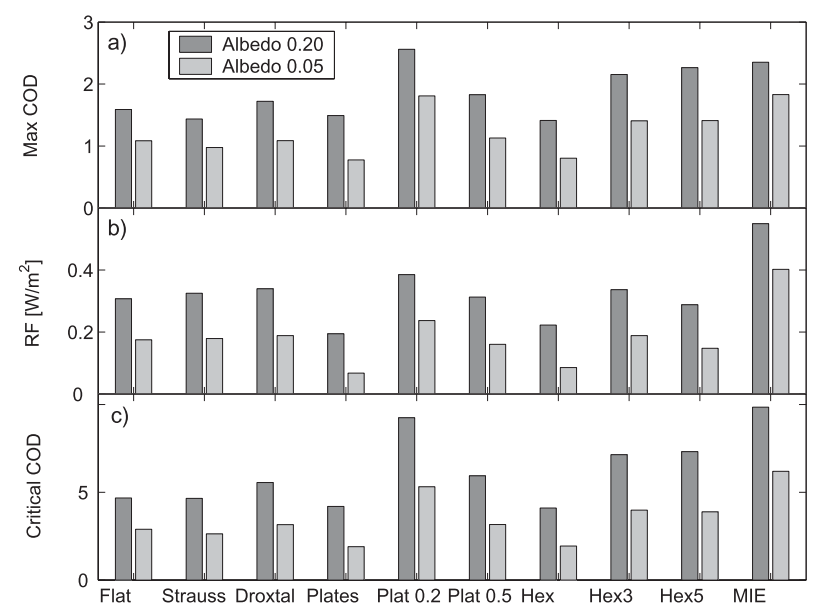

FIG. 13. (a) Contrail optical depth for which mean diurnal radiative forcing has a maximum, (b) maximum radiative forcing, and (c) COD for which mean diurnal radiative forcing is zero as a function of the crystal optical model. Lighter bars indicate cases with a surface albedo of 0.05 , and darker bars are for a surface albedo of 0.2 . The diurnal variability of radiative forcing was simulated for a latitude of $50^{\circ}$ and June conditions. 
columns with aspect ratios of 3 and 5, and spheres. In addition, optical properties of droxtals, hexagonal plates, and hexagonal columns derived by Yang et al. (2000, 2005) are used. Also, the Strauss et al. (1997) contrails model and a flat-optics model are included in the investigation. This gives in total 10 contrail models with various microphysical and optical properties. We found that, despite the fact that crystals are randomly oriented, there is a significant variation in the SSA and the asymmetry parameter among different crystal habits. The differences in these parameters reach up to 0.1. Both the crystal geometry and aspect ratio influence the optical properties of randomly oriented particles.

The SW and LW TOA contrail radiative forcings simulated with the MODTRAN code show significant variability with respect to the crystal shape. We found that the differences (relative to the mean values) in the SW, LW, and net radiative forcings among optical models reach $44 \%, 23 \%$, and $50 \%$, respectively. The smallest net forcings are obtained for the hexagonal columns (model 5) and hexagonal plates (model 4) models, whereas the spheres (model 10), Strauss (model 2 ), and droxtals (model 3) produce the largest net forcing values. This shape dependence of the radiative forcing might be particularly relevant from the contrail temporal-evolution point of view. Young contrails consist mostly of spherelike particles (Schröder et al. 2000) and therefore have stronger impact on climate than the more mature contrails, which have crystals with more irregular shapes (Goodman et al. 1998). This effect, however, might be somehow compensated or surpassed by an increase in optical depth, as a result of condensation of ambient humidity and an increase in ice water content.

The spherical particles, as already mentioned, produce the highest net forcing. Any departure from sphericity leads to lower forcing values, partly because particles with irregular shapes have higher albedo because of a smaller forward-scattering peak in the phase function. Indeed, an increase in contrail/cirrus albedo for nonspherical crystals has been reported in previous studies, both observational (Yang et al. 2010) and those based on modeling results (Penner et al. 1999; Zhang et al. 1999). It is interesting, however, to take a closer look at the impact of various aspect ratios on the SW radiative forcing. Our simulations indicate that from all investigated shapes the particles with the most extreme aspect ratios (plates: 0.2 and columns: 5 ), and therefore far away from sphericity, have the most similar SW forcing values to that of the spheres. Therefore the contrail models with these highly aspherical particles and the spheres have the most comparable albedos. On the other hand, Yang et al. (2010) pointed out, on the basis of the analysis of modeling results of Baum et al. (2005) and in situ measurements by Stackhouse and Stephens (1991), that the assumption of spherical ice crystals leads to underestimation of ice cloud albedo. Their result was particularly relevant for clouds with effective particle sizes of lower than $60 \mu \mathrm{m}$, which corresponds closely to the particle sizes within contrails (Heymsfield et al. 2010). These results, along with our calculations, suggest that contrails do not consist of ice crystals with either very large or very small aspect ratios. More observations are required to confirm these expectations.

The optical depths at which the diurnally averaged net radiative forcings reach their maximum (upper limit of warming) also exhibit significant variation with crystal habits. This parameter changes from 1.5 to 2.6 for a surface albedo of 0.2 and from 0.8 to 1.9 for a surface albedo of 0.05 . For comparison, typical contrails have optical depths between 0.05 and 0.5 (Kärcher et al. 2009). This indicates that an intensification of air traffic and a potential increase in the average optical depth of overlapping contrails will lead, globally, to a stronger warming of the earth's climate. Local effects, however, can have different magnitude and even a different sign depending on the external conditions and the assumed optical properties.

Sensitivity studies of the diurnally averaged contrail radiative forcings show that the critical optical depths at which the net forcings become negative range from 4.5 to 10 (for a surface albedo of 0.2 ) and 2 to 6.5 (for a surface albedo of 0.05 ). Only some aged-contrail cirrus clouds or aviation-induced cloudiness can possibly exceed such large optical depths. As a result of an increased negative SW forcing at larger solar zenith angles (Stuber et al. 2006; Myhre and Stordal 2001; see also Fig. 12), however, the instantaneous net forcing becomes negative for almost all analyzed crystal models. Such conditions, in turn, could potentially lead to temporarily negative net forcings. This suggestion agrees with the modeling study by Rap et al. (2010) who found, using the Met Office Climate Model, negative daytime-averaged net forcing values for latitudes between $45^{\circ}$ and $60^{\circ} \mathrm{N}$. In addition, they found a considerable frequency of negative net forcings (daily averaged) at the polar latitudes. These results indicate that regionally and under very specific scenarios contrails might be cooling the climate. From a climatological perspective, however, the cooling scenario is highly unlikely.

Acknowledgments. This study was funded by the EU FP6 project QUANTIFY (Contract 003893) and by the Polish Ministry of Higher Education and Science Grant 31/6.PR UE/2006/7. We thank Piotr Flatau from UCSD for many valuable discussions regarding the DDSCAT code and Andreas Macke for making the ray-tracing 
method available. We also acknowledge Ping Yang and coauthors for providing databases of optical properties of various crystal habits.

\section{REFERENCES}

Baum, B. A., A. J. Heymsfield, P. Yang, and S. T. Bedka, 2005: Bulk scattering models for the remote sensing of ice clouds. Part I: Microphysical data and models. J. Appl. Meteor., 44, 1885-1895.

Berk, A., L. S. Bernstein, G. P. Anderson, P. K. Acharya, D. C. Robertson, J. H. Chetwynd, and S. M. Adler-Golden, 1998: MODTRAN cloud and multiple scattering upgrades with application to AVIRIS. Remote Sens. Environ., 65, 367-375.

Bohren, C. F., and D. R. Huffmann, 1983: Absorption and Scattering of Light by Small Particles. Wiley-Interscience, $541 \mathrm{pp}$.

Dietmüller, S., M. Ponater, R. Sausen, K. P. Hoinka, and S. Pechtl, 2008: Contrails, natural clouds, and diurnal temperature range. J. Climate, 21, 5061-5075.

Draine, B. T., and P. J. Flatau, 1994: Discrete-dipole approximation for scattering calculations. J. Opt. Soc. Amer. A, 11, 1491-1499.

$\longrightarrow$, and $\longrightarrow$ 2003: User guide to the discrete dipole approximation Code DDSCAT.6.0. [Available online at http://arxiv. org/abs/astro-ph/0309069.]

Evans, K., and G. L. Stephens, 1995: Microwave radiative transfer trough clouds composed of realistically shaped ice crystals. Part I: Single scattering properties. J. Atmos. Sci., 52, 20412057.

Fortuin, J. P. F., R. van Dorland, W. M. F. Wauben, and H. Kelder, 1995: Greenhouse effects of aircraft emissions as calculated by a radiative transfer model. Ann. Geophys., 13, 413-418.

Fouquart, Y., B. Bonnel, and V. Ramaswamy, 1991: Intercomparing shortwave radiation codes for climate studies. J. Geophys. Res., 96, 8955-8968.

Frömming, C., M. Ponater, U. Burkhardt, A. Stenke, S. Pechtl, and R. Sausen, 2011: Sensitivity of contrail coverage and contrail radiative forcing to selected key parameters. Atmos. Environ., 45, 1483-1490.

Fu, Q., and K.-N. Liou, 1993: Parameterization of the radiative properties of cirrus clouds. J. Atmos. Sci., 50, 2008-2025.

Goodman, J., R. F. Pueschel, E. J. Jensen, S. Verma, G. V. Ferry, S. D. Howard, S. A. Kinne, and D. Baumgardner, 1998: Shape and size of contrails ice particles. Geophys. Res. Lett., 25, 1327-1330.

Halthore, R. N., and Coauthors, 2005: Intercomparison of shortwave radiative transfer codes and measurements. J. Geophys. Res., 110, D11206, doi:10.1029/2004JD005293.

Haywood, J. M., and Coauthors, 2009: A case study of the radiative forcing of persistent contrails evolving into contrailinduced cirrus. J. Geophys. Res., 114, D24201, doi:10.1029/ 2009JD012650.

Heymsfield, A. J., and C. M. R. Platt, 1984: A parameterization of the particle size spectrum of ice clouds in terms of the ambient temperature and the ice water content. J. Atmos. Sci., 41, 846-855.

, D. Baumgardner, P. DeMott, P. Forster, K. Gierens, and B. Kärcher, 2010: Contrail microphysics. Bull. Amer. Meteor. Soc., 91, 465-472.

Hong, G., 2007: Parameterization of scattering and absorption properties of nonspherical ice crystals at microwave frequencies. J. Geophys. Res., 112, D11208, doi:10.1029/ 2006JD008364.
—, Q. Feng, P. Yang, G. Kattawar, P. Minnis, and Y. Hu, 2008: Optical properties of ice particles in young contrails. J. Quant. Spectrosc. Radiat. Transfer, 109, 2635-2657.

Jensen, E. J., S. Kinne, and O. B. Toon, 1994: Tropical cirrus cloud radiative forcing: Sensitivity studies. Geophys. Res. Lett., 21, 2023-2026.

Kahnert, M., A. D. Sandvik, M. Biryulina, J. J. Stamnes, and K. Stamnes, 2008: Impact of ice particle shape on short-wave radiative forcing: A case study for an arctic ice cloud. J. Quant. Spectrosc. Radiat. Transfer, 109, 1196-1218.

Kärcher, B., O. Möhler, P. J. DeMott, S. Pechtl, and F. Yu, 2007: Atmospheric chemistry and physics insights into the role of soot aerosols in cirrus cloud formation. Atmos. Chem. Phys., 7, 4203-4227.

— U. U. Burkhardt, S. Unterstrasser, and P. Minnis, 2009: Factors controlling contrail cirrus optical depth. Atmos. Chem. Phys., 9, 6229-6254.

,-- M. Ponater, and C. Frömming, 2010: Importance of representing optical depth variability for estimates of global line-shaped contrail radiative forcing. Proc. Natl. Acad. Sci. USA, 107, 19 181-19 184, doi:10.1073/pnas.1005555107.

Kim, M.-J., 2006: Single scattering parameters of randomly oriented snow particles at microwave frequencies. J. Geophys. Res., 111, D14201, doi:10.1029/2005JD006892.

Liou, K. N., 1992: Radiation and Cloud Processes in the Atmosphere. Oxford University Press, $487 \mathrm{pp}$.

Liu, G., 2008: A database of microwave single-scattering properties for nonspherical ice particles. Bull. Amer. Meteor. Soc., 89, 1563-1570.

Macke, A., and M. I. Mishchenko, 1996: Applicability of regular particle shapes in light scattering calculations for atmospheric ice particles. Appl. Opt., 35, 4291-4296.

_ _ J. Muller, and E. Rasche, 1996: Single scattering properties of atmospheric crystals. J. Atmos. Sci., 53, 2813-2825.

Marquart, S., M. Ponater, F. Mager, and R. Sausen, 2003: Future development of contrail cover, optical depth, and radiative forcing: Impacts of increasing air traffic and climate change. J. Climate, 16, 2890-2904.

Meerkötter, R., U. Schumann, D. R. Doelling, P. Minnis, T. Nakajima, and Y. Tsushima, 1999: Radiative forcing by contrails. Ann. Geophys., 17, 1070-1084.

Meyer, R., H. Mannstein, R. Meerkötter, U. Schumann, and P. Wendling, 2002: Regional radiative forcing by line-shaped contrails derived from satellite data. J. Geophys. Res., 107, 4104, doi:10.1029/2001JD000426.

Minnis, P., U. Schumann, D. R. Doelling, K. M. Gierens, and D. W. Fahey, 1999: Global distribution of contrail radiative forcing. Geophys. Res. Lett., 26, 1853-1856.

Mishchenko, M. I., and L. D. Travis, 1994: T-matrix computations of light scattering by large spheroidal particles. Opt. Commun., 109, 16-21.

Myhre, G., and F. Stordal, 2001: On the tradeoff of the solar and thermal infrared radiative impact of contrails. Geophys. Res. Lett., 28, 3119-3122.

— , and Coauthors, 2009: Intercomparison of radiative forcing calculations of stratospheric water vapour and contrails. Meteor. Z., 18, 585-596.

Palikonda, R., P. Minnis, D. P. Duda, and H. Mannstein, 2005: Contrail coverage derived from 2001 AVHRR data over the continental United States of America and surrounding areas. Meteor. Z., 14, 525-536.

Penner, J. E., D. H. Lister, D. J. Griggs, D. J. Dokken, and M. McFarland, Eds., 1999: Aviation and the Global Atmosphere. Cambridge University Press, 365 pp. 
Ponater, M., S. Marquart, and R. Sausen, 2002: Contrails in a comprehensive global climate model: Parametrization and radiative forcing results. J. Geophys. Res., 107, 4164, doi:10.1029/ 2001JD000429.

Rädel, G., and K. P. Shine, 2008: Radiative forcing by persistent contrails and its dependence on cruise altitudes. J. Geophys. Res., 113, D07105, doi:10.1029/2007JD009117.

Rap, A., P. M. Forster, A. Jones, O. Boucher, J. M. Haywood, N. Bellouin, and R. R. De Leon, 2010: Parameterization of contrails in the UK Met Office Climate Model. J. Geophys. Res., 115, D10205, doi:10.1029/2009JD012443.

Rockwitz, K., 1989: Scattering properties of horizontally oriented ice crystal columns in cirrus clouds. Part 1. Appl. Opt., 28, 4103-4110.

Sausen, R., and Coauthors, 2005: Aviation radiative forcing in 2000: An update on IPCC (1999). Meteor. Z., 14, 555-561.

Schröder, F., and Coauthors, 2000: On the transition of contrails into cirrus clouds. J. Atmos. Sci., 57, 464-480.

Solomon, S., D. Qin, M. Manning, M. Marquis, K. Averyt, M. M. B. Tignor, H. L. Miller Jr., and Z. Chen, Eds., 2007: Climate Change 2007: The Physical Science Basis. Cambridge University Press, 996 pp.

Stackhouse, P. W., Jr., and G. L. Stephens, 1991: A theoretical and observational study of the radiative properties of cirrus clouds: Results from FIRE 1986. J. Atmos. Sci., 48, 2044-2059.

Stamnes, K., S. Tsay, W. Wiscombe, and K. Jayaweera, 1988: Numerically stable algorithm for discrete-ordinate-method radiative transfer in multiple scattering and emitting layered media. Appl. Opt., 27, 2502-2509.
Strauss, B., R. Meerkötter, B. Wissinger, P. Wendling, and M. Hess, 1997: On the regional climatic impact of contrails: Microphysical and radiative properties of contrails and natural cirrus clouds. Ann. Geophys., 15, 1457-1467.

Stuber, N., P. Forster, G. Rädel, and K. Shine, 2006: The importance of the diurnal and annual cycle of air traffic for contrail radiative forcing. Nature, 441, 864-867.

Sun, W., Q. Fu, and Z. Chen, 1999: Finite-difference time-domain solution of light scattering by dielectric particles with a perfectly matched layer absorbing boundary condition. Appl. Opt., 38, 3141-3151.

Yang, P., and K. N. Liou, 1996: Finite-difference time domain method for light scattering by small ice crystals in three-dimensional space. J. Opt. Soc. Amer. A, 13, 2072-2085.

,,-- K. Wyser, and D. Mitchell, 2000: Parameterization of the scattering and absorption properties of individual ice crystals. J. Geophys. Res., 105, 4699-4718.

—, H. Wei, H.-L. Huang, B. A. Baum, Y. X. Hu, G. W. Kattawar, M. I. Mishchenko, and Q. Fu, 2005: Scattering and absorption property database for nonspherical ice particles in the nearthrough far-infrared spectral region. Appl. Opt., 44, 55125523.

, G. Hong, A. E. Dessler, S. C. Ou, K.-N. Liou, P. Minnis, and Harshvardhan, 2010: Contrails and induced cirrus: Optics and radiation. Bull. Amer. Meteor. Soc., 91, 473-478.

Zhang, Y., A. Macke, and F. Albers, 1999: Effect of crystal size spectrum and crystal shape on stratiform cirrus radiative forcing. Atmos. Res., 52, 59-75. 